\title{
Why Does General QoL Tend to Worsen Over Time Following Septoplasty Even in the Absence of Worsening Nasal Symptoms?
}

\author{
${\text { Elena } \text { Cantone }^{1} \text { (D) } \cdot \text { Maurizio Iengo }}^{1}$
}

Received: 30 August 2020/Accepted: 5 October 2020/Published online: 15 October 2020

(C) The Author(s) 2020

\begin{abstract}
Septoplasty is one of the most frequent surgical procedures performed by head and neck surgeons. Despite surgical correction, some patients are not satisfied with their postoperative outcomes. Only a few papers report data on the long-term quality of life of patients after septoplasty, and none over 36 months. This follow up study on 68 surgical procedures aims to evaluate the differences between short term and long-term quality of life after septoplasty using a disease specific quality of life questionnaire, the NOSE, and a general health status questionnaire, the SF-36. We found a statistically significant decrease of the total long-term SF-36 scores, but we did not find changes of the NOSE scores compared with the shortterm scores. These results indicated that patients perceived a worsening of their quality of life after more than 36 months following septoplasty, even in the absence of specific nasal symptoms. We could speculate that the preoperative general health performance was erroneously attributed to specific nasal symptoms, probably incorrectly emphasized or that patients did not correctly remember the preoperative clinical status. This research pointed out the importance of patients' selection and of a thorough evaluation of their preoperative expectations before surgical planning.
\end{abstract}

Keywords Septoplasty - Symptoms · Quality of life · Questionnaires · NOSE · SF-36

Elena Cantone

cantoneent@gmail.com

1 Department of Neuroscience, ENT section, "Federico II" University of Naples, Via Pansini, 5, Naples, Italy

\section{Introduction}

Nasal obstruction is one the most common symptoms observed in head and neck clinical practice. It is directly related to the quality of life $(\mathrm{QoL})$ and frequently caused by septal deviation. Septoplasty represents one of the most frequent surgical procedures performed by head and neck surgeons. However, despite surgical correction, some patients are not satisfied with their postoperative outcomes with consequent impairment of QoL and medicolegal problems [1-3].

Sometimes, patients complaining significant postoperative nasal obstruction may have a small residual septal deviation, whereas patients complaining mild symptoms may have a severe anatomical deformity [4]. These data suggest the presence of additional factors, independent of surgical outcomes, responsible for impaired QoL following septoplasty.

So far, only a few papers exist reporting data on the impact of septoplasty on long-term QoL [5].

The aim of this study was to evaluate the severity of long-term (after 36 months or more) symptoms following septoplasty and their impact on QoL compared with shortterm scores.

\section{Material and Methods}

This follow up study included primary septoplasties with turbinoplasty performed at ENT Departments of the "Federico II" University with a follow-up time of at least 36 months.

Patients gave their written informed consent to the study, which was fully approved by the Board of Medical Ethics of University of Naples "Federico II" (n.113/20). 
The study was carried out according to the Declaration of Helsinki.

All patients were identified in hospital records. Medical charts were searched for: date of surgery, gender, age, primary surgery or reoperation, surgical technique, history of nasal trauma or allergy, preoperative nasal endoscopy (NE) and active anterior rhinomanometry (AAR) [6].

Exclusion criteria were: age $<18$ years, previous nasal surgery, trauma, chronic rhinosinusitis, allergy, diabetes, a history of radiation therapy to the head and neck, immunologic, heart, oncologic, malformative and neuropsychiatric diseases.

Follow up data were retrieved from the outpatient medical reports. We compared postoperative symptom relief at 6 months (T1) with that at 36-55 months (T2) using two different QoL questionnaires: the Nasal Obstruction Symptoms Evaluation (NOSE) questionnaire and the 36-Item Short Form Health Survey (SF-36) questionnaire.

The NOSE assessed disease specific symptoms, i.e. nasal obstruction, using a 4-points grading with higher scores meaning more severe nasal symptoms [7]. SF-36 questionnaire measured patients' general health status in the previous month. It contains 36 questions that represent eight health concepts by grouping into subgroups-physical functioning (PF), role-physical (RP), role emotional (RE), bodily pain (BP), general health (GH), vitality (VT), social functioning (SF) and mental health (MH). One question was related to the change of the patient's health status in the previous year.

Results of SF-36 were expressed as percentages and the higher the result, the better the patient's general health [8].

During outpatient follow up subjects were also asked to report the onset of new pathologies or events that could have altered their QoL, in these cases they were excluded from the study.

\section{Statistical Analysis}

The SPSS-PC, (version 16; SPSS Inc., Chicago, IL) was used for statistical analyzes. The Pearson's correlation was used to determine the correlation between two numerical variables. The t-Student test was used to evaluate differences in the nasal obstruction metrics at $\mathrm{T} 1$ and $\mathrm{T} 2$. A $p$ value of $<0.05$ was considered statistically significant.

\section{Results}

Sixty-eight patients [43 M, $25 \mathrm{~F}$; age range 18-54 years, $28.9 \pm 9.6$ (mean $\pm \mathrm{SD}$ )] met criteria for inclusion, mean follow up $44.05 \pm 5.9$ months. According to the medical records, before surgery the diagnosis of nasal obstruction had been confirmed by NE with a 4-mm 30-degree rigid endoscope (Storz, Tuttlingen, Germany) and AAR (Diagnostic cube, Rhino 31 Atmos Medizintechnik Lenzkirch, Germany) performed before and after decongestion under the same standard conditions, in accordance with the International Standardization Committee for Rhinomanometry [6].

Preoperative NE and AAR [total nose resistance (TNR) $0.49 \pm 0.19$ (mean $\pm \mathrm{SD}$ )] confirmed the nasal obstruction due to septal deviation. Preoperative NOSE was $51.20 \pm 14$ (mean \pm SD) and SF-36 41.54\%. Surgical procedures were performed under general anesthesia by two experienced surgeons that decided and performed the operations.

Septoplasties were performed using the maxilla-premaxilla approach including a hemi-transfixion incision followed by an elevation of the septal mucoperichondrium in one side, combining mobilization, reshaping and/or removing the deviated part of the cartilage and/or by reconstruction of the septum support [5].

We performed submucous inferior turbinoplasty (without bony resection) and out-fracture of inferior turbinates [9].

We did not find statistically significant differences $(p=0.75)$ between T1 and T2 NOSE (Table 1).

We did not find statistically significant differences in the $\mathrm{T} 1$ versus T2 NOSE in males (T1NOSE $21.5 \pm 20.2$ vs. T2NOSE $21.7 \pm 17.6 ; p=0.95$ ) and females (T1NOSE $20.6 \pm 20.4$ vs. T2NOSE $23.2 \pm 23.6 ; p=0.70$ ) or between males and females (T1 NOSE males $21.5 \pm 20.2$ vs. T1NOSE females $20.6 \pm 20.4 ; p=0.85-\mathrm{T} 2$ NOSE males $21.7 \pm 17.6$ vs. T2NOSE females $23.2 \pm 23.6$; $p=0.77)$.

We did not find correlation between NOSE and age neither at T1 $(p=0.11)$ nor at T2 $(p=0.12)$.

$\mathrm{T} 1$ versus $\mathrm{T} 2 \mathrm{SF}-36$ scores demonstrated a statistically significant decrease $(p=0.0001)$ of percentage in the longterm follow up (Table 1).

The SF-36 scores T1 versus T2 in males (T1 SF-36 $55.4 \pm 4.2$ vs. T2 SF-36 $68.3 \pm 3.8 ; p=0.0001)$ and females (T1 SF-36 $55 \pm 5.2$ vs. T2SF-36 $67 \pm 2.8$; $p=0.0001$ ) showed statistically significant differences, whereas SF-36 scores between males and females at T1 (T1 SF-36 males $55.4 \pm 4.2$ vs. T1SF-36 females $55 \pm 5.2 ; p=0.73)$ and at T2 (T2 SF-36 males $68 \pm 3.8$ vs. T2 SF-36 females $67 \pm 2.8 ; p=0.5$ ) did not show statistically significant gender differences.

There was no correlation between SF-36 and age [T1 $(p=0.9)$; T2 $(p=0.4)]$ and no correlation between NOSE and SF-36 [T1 $(p=0.2)$ and T2 $(p=0.06)]$. SF-36 subscales scores at $\mathrm{T} 1$ were higher than at $\mathrm{T} 2(p=0.0001)$, with most compromised subscales represented by BP 
Table 1 The comparison T1 versus T2 NOSE and SF-36 scores demonstrated a statistically significant decrease of the total SF-36 score alone in long term follow up (mean $\pm \mathrm{SD})$

\begin{tabular}{llll}
\hline & T1 & T2 & $p$ \\
\hline NOSE & $21.18 \pm 20.1$ & $22.2 \pm 19.9$ & 0.75 \\
SF-36 & $82.4 \pm 6.9$ & $67.9 \pm 3.5$ & $0.0001^{*}$ \\
\hline
\end{tabular}

* represents statistically significant

$(p<0.05), \quad \mathrm{GH} \quad(p<0.05), \quad \mathrm{VT} \quad(p<0.05)$, and $\mathrm{SF}$ $(p<0.05)$ (Fig. 1).

\section{Discussion}

Sometimes after a septoplasty the deviation can persist. Surprisingly, some persistent septal deviations are associated with patient's satisfaction and good QoL, whereas completely restored deviations are not $[1,5]$

Although previous studies have already investigated assessment, outcomes, clinical implications and patients' satisfaction after primary septoplasty, most publications suffered from various limitations in design. Many of them were based on different surgical techniques, used only telephone follow-up surveys, relied on physician-rated outcomes rather than patient assessments of outcome, or applied either not validated outcome measures or validated outcome measures not specifically designed for nasal obstruction. In addition, previous research was based on postoperative evaluation only in the short-term or, in any case, no longer than 36 months $[1,4,5]$.

Since patients, in general, tend to rate the results of their septal surgery less positively as the postoperative period gets longer, it is mandatory to evaluate surgical outcomes in the long-term [5].

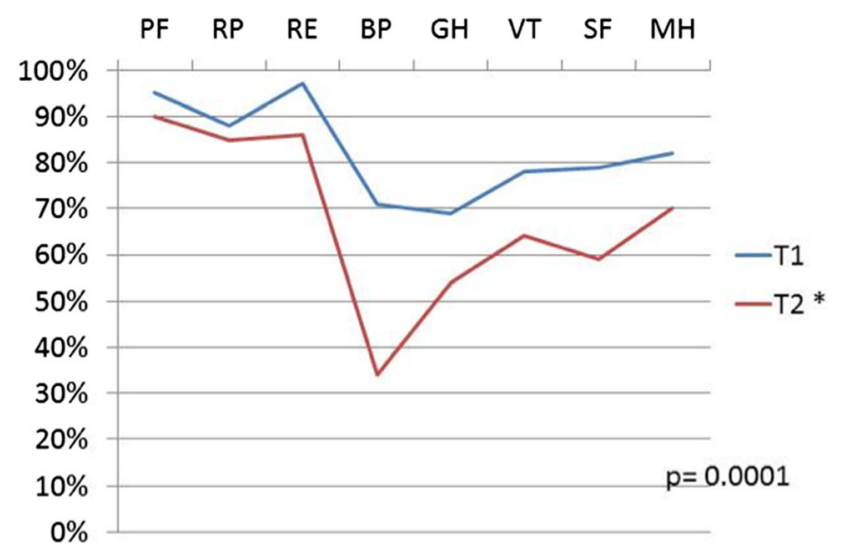

Fig. 1 Short Form-36 (SF-36) subscores test results (\%). SF-36 subscales scores at T1 were higher than at T2; *0.0001. Physical functioning (PF), role-physical (RP), role-emotional (RE), bodily pain (BP), general health (GH), vitality (VT), social functioning (SF) and mental health $(\mathrm{MH})$
Our study aims at examining the postoperative outcomes more than 36 months after septoplasty in terms of subjective assessment with the use of two QoL questionnaires, the first specifically designed for nasal symptoms (NOSE) and the second evaluating the patients' general health status (SF-36).

We found no correlation between age at the time of surgery as well as gender, and questionnaires' scores. Furthermore, we did not find correlation between NOSE and SF-36 scores. We found a statistically significant worsening of the total general health SF-36 score at T2 compared with SF-36 score at T1 follow up (Table 1). We also found that results were generally better among all of the eight test subgroups at short-term $\mathrm{T} 1$ follow up than at long-term T2 follow up (Fig. 1).

In our sample size, while the specific nasal symptomatology assessed by NOSE did not change over time, the general health status assessed by SF-36 did. These results showed that the general QoL expressed by SF-36, was perceived as worsened over time, despite the stability of the nasal-specific symptoms, as indicated by NOSE. In particular, among SF-36 subscales the most altered ones at T2 were BP, GH, VT, and SF. On the one hand, for GH, VT, SF we could think that the passing of time and the increasing age affect these subscales, on the other, for BP an explanation is rather difficult to provide.

We might suppose that the preoperative general health performance was erroneously attributed to specific nasal symptoms that were, probably, wrongly emphasized.

It must be also considered the possibility that patients did not correctly remember the preoperative clinical status.

Despite many differences among studies, all researchers showed an unsatisfactory QoL after septoplasty [10].

For this reason, it is crucial the patients' selection and dealing with the patient' expectations; the surgeon should make it clear to the patient as much as possible which symptoms can resolve and which cannot. Indeed, very often, the patient's priorities are different from the surgeon's clinical assessment. In addition, some patients could erroneously assume that any surgery will cure even not mentioned symptoms.

However, our study, demonstrated that QoL rate after septoplasty decreased with time, even after 55 months $[3,10]$. 


\section{Conclusions}

Our research showed that general QoL, assessed by SF-36, worsened over time, despite NOSE indicated that the nasalspecific symptoms did not. So, it is of utmost importance the patients' selection and especially the thorough evaluation of their preoperative expectations before surgical planning.

Likewise, it is important to keep patients in a long-time postoperative follow up.

Author Contributions EC contributed to conceptualization, data curation, formal analysis, investigation, methodology, writingoriginal draft, writing-review and editing; MI contributed to supervision, validation, visualization, writing — review and editing.

Funding Open access funding provided by Università degli Studi di Napoli Federico II within the CRUI-CARE Agreement.

\section{Compliance with Ethical Standards}

Conflict of interest The authors declare that there is no conflict of interest.

Ethical Approval All procedures performed in studies involving human participants were in accordance with the ethical standards of the institutional and/or national research committee and with the 1964 Helsinki declaration and its later amendments or comparable ethical standards.

Open Access This article is licensed under a Creative Commons Attribution 4.0 International License, which permits use, sharing, adaptation, distribution and reproduction in any medium or format, as long as you give appropriate credit to the original author(s) and the source, provide a link to the Creative Commons licence, and indicate if changes were made. The images or other third party material in this article are included in the article's Creative Commons licence, unless indicated otherwise in a credit line to the material. If material is not included in the article's Creative Commons licence and your intended use is not permitted by statutory regulation or exceeds the permitted use, you will need to obtain permission directly from the copyright holder. To view a copy of this licence, visit http://creativecommons.org/licenses/by/4.0/.

\section{References}

1. Kuduban O, Bingol F, Budak A et al (2015) The reason of dissatisfaction of patient after septoplasty. Eur J Med 47:190-193

2. Gamerra M, Cantone E, Sorrentino G et al (2017) Mathematical model for the preoperative identification of obstructed nasal subsites. Acta Otorhinolaryngol Ital 37:410-415

3. Cantone E, Ricciardiello F, Oliva F et al (2018) Septoplasty: Is it possible to identify potential "predictors" of surgical success? Acta Otorhinolaryngol Ital 36:528-535

4. Konstantinidis I, Triaridis S, Triaridis A et al (2005) Long term results following nasal septal surgery. Focus on patients' satisfaction. Auris Nasus Larynx 32:369-374

5. Valsamidis K, Titelis K, Rachovitsas D et al (2018) Long-term evaluation of nasal septoplasty followed by inferior turbinate cauterization for the treatment of nasal obstruction using objective and subjective methods. Int Arch Otorhinolaryngol 22:284-290

6. Clement PA (1997) Rhinomanometry. Allergy 52(33 Suppl):26-27

7. Dolan RW (2010) Minimally invasive nasal valve repair, an evaluation using the NOSE scale. Arch Otolaryngol Head Neck Surg 36:292-295

8. Shejbal D, Vagić D, Stevanović S et al (2012) Impact of posttraumatic stress disorder on sinonasal symptoms and quality of life in patients with chronic rhinosinusitis. Patient Prefer Adherence 6:847-852

9. Sulsenti G, Palma P (1996) Tailored nasal surgery for normalization of nasal resistance. Facial Plast Surg 12:333-345

10. Sundh C, Sunnergren O (2015) Long-term symptom relief after septoplasty. Eur Arch Otorhinolaryngol 272:2871-2875

Publisher's Note Springer Nature remains neutral with regard to jurisdictional claims in published maps and institutional affiliations. 